\title{
Is hemifacial spasm accompanied by hemodynamic changes detectable by ultrasound?
}

\author{
Fabienne Perren • Michel R. Magistris
}

Received: 30 July 2013 / Accepted: 8 May 2014 / Published online: 12 June 2014

(C) Springer-Verlag Wien 2014

\begin{abstract}
Background Arterial tortuosity of the posterior circulation compressing the facial nerve induces the ephaptic axonoaxonal cross-talk that sparks hemifacial spasm. We sought if a noninvasive method such as color duplex of these arteries might detect hemodynamical changes in this condition.

Methods Nine patients with hemifacial spasm, successfully treated with botulinum toxin, were examined with color-coded duplex ultrasound. Mean blood flow velocities of the vertebral, basilar, posterior inferior cerebellar, and anterior inferior cerebellar arteries were measured and side-to-side comparison performed. Results In all nine patients, the mean blood flow velocity, averaging across the two arteries, was higher on the side of the hemifacial spasm (Fisher's exact $p<0.008$; two-tailed). The results of the repeated measures ANOVA show that the main effect of side of flow was statistically significant, $\mathrm{F}(1,8)=17.354, p=.0032$, with higher mean blood flow velocities observed on the side of the hemifacial spasm. There was no significant association between the mean flow velocity of the vertebral artery and the side of $\operatorname{spasm}(p=0.523)$.

Conclusions Hemifacial spasm also seems to relate to hemodynamic changes, which may be detectable by color duplex imaging.
\end{abstract}

F. Perren $(\bowtie) \cdot M$. R. Magistris

Division of Neurology, Department of Clinical Neurosciences, HUG, University Hospital and Medical Faculty of Geneva, Rue Gabrielle-Perret-Gentil 4, 1211 Geneva 14, Switzerland

e-mail: fabienne.perren@hcuge.ch

F. Perren

Neurosonology and Neurovascular Unit, Department of Clinical Neurosciences, HUG, University Hospital and Medical Faculty of Geneva, Geneva, Switzerland

\section{R. Magistris}

Electroneuromyography Unit \& Neuromuscular Disorders, HUG, University Hospital and Medical Faculty of Geneva, Geneva, Switzerland
Keywords Hemifacial spasm · Color-coded duplex ultrasound $\cdot$ Hemodynamics $\cdot$ Facial nerve $\cdot$ Posterior circulation

\section{Introduction}

Hemifacial spasm (HFS) is an infrequent condition with a reported incidence of 0.8 per 10,000 per year with a male-tofemale ratio of $35: 65$ [1, 2]. It is characterized by intermittent, painless, involuntary spasmodic contractions of the muscles innervated by the facial nerve on one side of the face. Babinski reported as an early and specific sign of HFS, closure of the eye on the side of the spasm when the patient raises the ipsilateral eyebrow [3]. It affects typically the middle-age population and, according to some authors, the left side seems more often affected than the right [4]. HFS is responsible for neurological disability with potentially severe esthetic and social impact on the patients' quality of life [5]. Generally, an arterial tortuosity or an aneurysm of the vertebrobasilar circulation compressing the facial nerve as it exits from brainstem induces the ephaptic axono-axonal cross-talk that sparks HFS. Rarely, it can be secondary to mass lesions such as cerebellopontine angle neurinomas, cholesteatomas, or meningiomas [5]. Diagnosis of typical HFS is based upon a clinical history of the reported symptoms, and an otherwise normal neurological and cranio-facial examination. Electroneurography may demonstrate the abnormal ephaptic axono-axonal connections that cause the synkinesis and the spasms [6]. Magnetic resonance imaging (MRI) may visualize compression of the facial nerve by a blood vessel or, rarely, by another cause. However, magnetic resonance angiography (MRA), the actual gold standard, does not always show the "vascular-nerve conflict". Although confident identification of the PICA may be difficult and failures to insonate of up to $50 \%$ have been reported [7], we sought if a noninvasive 
method such as color-coded duplex flow imaging (CDFI) of these arteries might detect hemodynamical changes in HFS. Specifically, we expected that there would be a systematic difference in hemodynamic parameters on the side of the HFS. In addition, we looked to see whether any such side difference might be more localized to the PICA versus the AICA or the VA.

\section{Methods}

Twelve consenting patients suffering from HFS, all successfully treated with botulinum toxin, were examined prospectively. CDFI (extra- and transcranial using 7.5-MHz and 2$\mathrm{MHz}$ devices) was performed by a sonographer who was blinded as to the side of the HFS. Vessel examination of the unaffected side served as the control. Blood flow velocities (peak systolic, peak diastolic, and mean flow velocities) of the vertebral (VA), basilar (BA), posterior inferior cerebellar (PICA) (Fig. 1) and anterior inferior cerebellar (AICA) arteries as well as the diameter of the VA in their intervertebral segment (V2) were measured and a side-to-side comparison was performed. Transcranial examination of the intracranial vessels was performed from a transnuchal approach (through the foramen magnum acoustic bone window). The PICA as a branch of the vertebral artery in its distal V4 segment (depth of $60-70 \mathrm{~mm}$ ) was detected as a flow signal heading initially laterally and towards the 2-MHz device. The AICA arises from the basilar artery just after the vertebrobasilar junction (depth $80-90 \mathrm{~mm}$ ) as a flow signal heading laterally towards the 2-MHz device (Fig. 2). Measurements of the blood flow velocities of the intracranial vessels were performed without angle correction. In order to exclude symptomatic etiologies (intracerebral lesions with mass effect) of the HFS, all the
Fig. 1 Color-coded duplex ultrasound imaging of the posterior inferior cerebellar artery (PICA) showing: the site of vessel examination and: the Doppler signal with measured mean blood flow velocities (Vm peak in $\mathrm{cm}$ / s) of the right (PICAR) compared to the left posterior inferior cerebellar artery (PICAL) of a patient suffering from right HFS

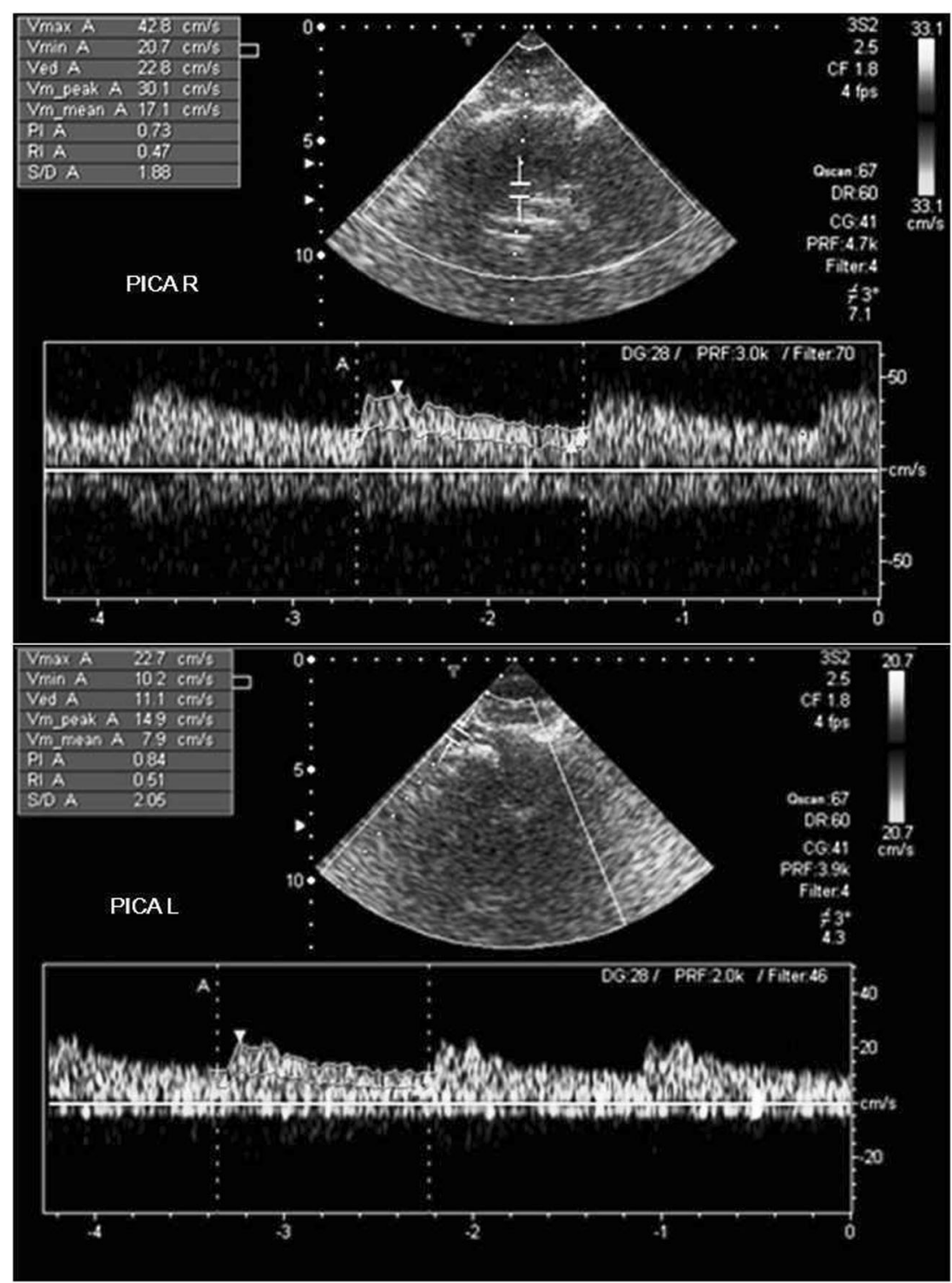


Fig. 2 Transnuchal color-coded duplex ultrasound examination of the posterior arterial circulation showing the vertebral arteries in their V4 segment (VA) and the posterior inferior cerebellar arteries (PICA) arising from them; and the anterior inferior cerebellar arteries (AICA) arising from the basilar artery (BA). $R$ right; $L$ left

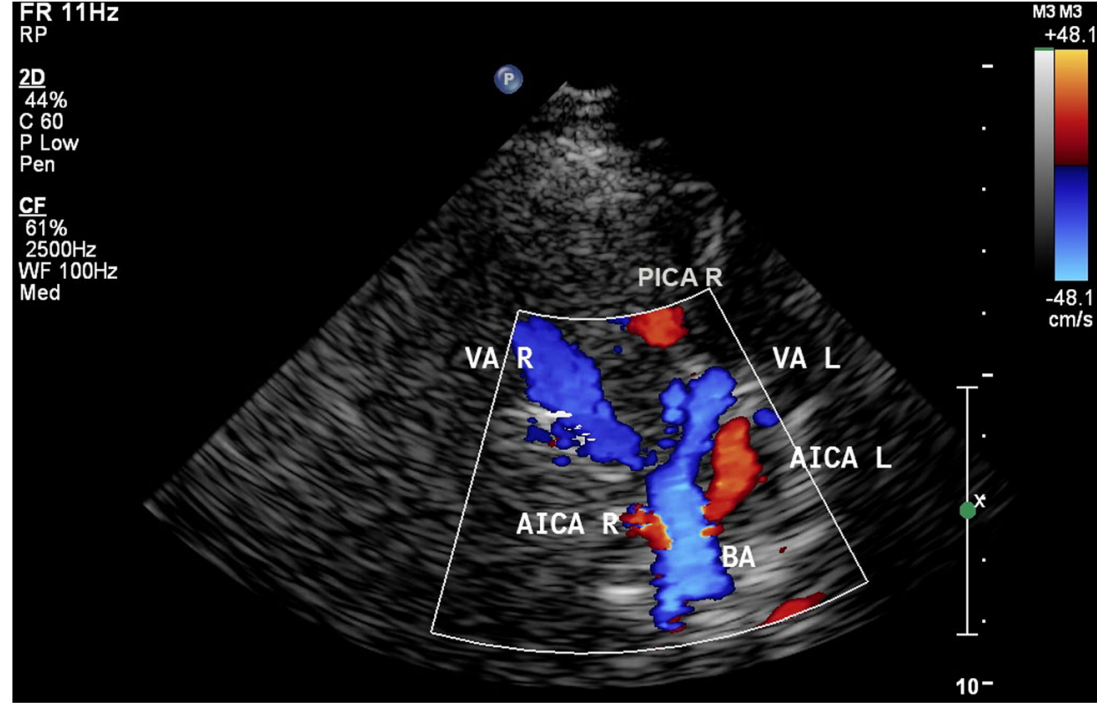

patients underwent MRI of the brain. Those suffering from stroke, intra- or extracranial vessel stenosis, dissection, sub- or occlusion were excluded. This study was approved by our institutional ethics committee and written informed patient consent to perform this study was received.

\section{Results}

Twelve patients (six men, six women; mean age, 61.6 years) were examined. In three of them, full examination of the intracranial vessels was not possible because of absent acoustic bone windows (no suitable site on the bone through which transcranial insonification can be performed, which can be found up to $20 \%$ ) leading to non-depiction of at least one AICA and PICA, not allowing for comparison of sides. Nine patients (five men, four women; mean age, 53.4 years) were fully studied. In all of them, the PICAs were arising from the
V4 segment of the VA. In four patients, injection of echocontrast agent was needed to depict all vessels on both sides. In all nine of the nine patients, the mean blood flow velocity (MFV), averaging across the two arteries (AICA and PICA), was higher on the side of the HFS (Fisher's exact $p<0.008$; two-tailed).

The results of the repeated measure ANOVA show that the main effect of side of flow was statistically significant, $\mathrm{F}(1,8)=17.354, p=.0032$, with higher mean blood flow velocities observed on the side of the hemifacial spasm. There was no systematic difference in this effect between the two arteries. Side by artery interaction, $\mathrm{F}(1,8)=0.106, p>.5$, indicating that neither artery was more likely to be the source of the side difference in this sample (Table 1).

There was no significant association between MFV of the VA (V0-4) and the side of HFS (Fisher's exact $p=0.523$ ). Mean diameters of the vertebral arteries, as measured with CDFI in their V2 segment, showed no VA hypoplasia

Table 1 Baseline data and comparison of the posterior inferior cerebellar arteries (PICA) and anterior inferior cerebellar arteries (AICA) mean flow velocities (MFV) and side of the hemifacial spasm (HFS)

\begin{tabular}{|c|c|c|c|c|c|c|c|c|c|c|c|}
\hline \multirow[t]{2}{*}{ Patient } & \multirow[t]{2}{*}{ Age (years) } & \multirow[t]{2}{*}{ Gender } & \multicolumn{2}{|c|}{ VA diameter (mm) } & \multicolumn{4}{|c|}{$\operatorname{MFV}(\mathrm{cm} / \mathrm{s})$} & \multicolumn{2}{|c|}{ RI (resistance index) } & \multirow[t]{2}{*}{ HFS } \\
\hline & & & Right & Left & PIC & ht/left & $\mathrm{AIC}$ & hht/left & PICA, right/left & AICA, right/left & \\
\hline 1. & 29 & M & 46 & 45 & 24 & 18 & 35 & 26 & $0.58 / 0.56$ & $0.55 / 0.53$ & Right \\
\hline 2. & 66 & $\mathrm{~F}$ & 44 & 55 & 34 & 13 & 31 & 37 & $0.60 / 0.59$ & $0.64 / 0.65$ & Right \\
\hline 3. & 41 & M & 41 & 38 & 18 & 46 & 17 & 41 & $0.42 / 0.53$ & $0.46 / 0.51$ & Left \\
\hline 4. & 53 & M & 39 & 42 & 30 & 14 & 12 & 11 & $0.47 / 0.51$ & $0.55 / 0.48$ & Right \\
\hline 5. & 59 & $\mathrm{~F}$ & 39 & 46 & 24 & 19 & 41 & 32 & $0.60 / 0.55$ & $0.54 / 0.52$ & Right \\
\hline 6. & 62 & $\mathrm{~F}$ & 44 & 34 & 25 & 37 & 22 & 46 & $0.69 / 0.70$ & $0.62 / 0.68$ & Left \\
\hline 7. & 76 & $\mathrm{~F}$ & 30 & 38 & 21 & 18 & 21 & 25 & $0.64 / 0.69$ & $0.65 / 0.62$ & Left \\
\hline 8. & 49 & M & 30 & 38 & 18 & 27 & 16 & 29 & $0.50 / 0.50$ & $0.59 / 0.53$ & Left \\
\hline 9. & 46 & M & 31 & 38 & 12 & 17 & 29 & 38 & $0.70 / 0.65$ & $0.62 / 0.61$ & Left \\
\hline
\end{tabular}


(diameter $\leq 20 \mathrm{~mm}$ ) and did not differ significantly between both sides: right VAs: $38 \pm 8 \mathrm{~mm}$; left VAs: $39 \pm 5 \mathrm{~mm}$.

\section{Discussion}

According to the literature, the compression of the facial nerve at its root exit zone is most often due to the AICA, which arises at the lowest third of the basilar artery followed, in order of frequency, by the PICA arising from the distal part of the vertebral artery $[5,8,9]$. Less frequently, the VA or multiple offenders are envisaged responsible for the HFS [8, 9]. Vessel diameter asymmetry is frequent in the posterior vertebrobasilar circulation and vessel diameter dominance, either of the AICA, PICA, or VA, seems to be associated with the side of HFS [8, 9]. However, due to the low frequency of insonation $(2 \mathrm{MHz})$, it is actually still not possible to measure the diameter of the intracranial vessels. Furthermore, arterial hypertension, which was found in 7/12 (58\%) of our patients, appears to be frequent $(67 \%)$ in patients with HFS [10]. Reference values of PICA blood flow velocities $(\mathrm{cm} / \mathrm{s})$ from a small series [7] are peak systolic: $48 \pm 5$; end-diastolic: $26 \pm 4$ but are still not available for the AICA.

Using a non-invasive dynamic measure, i.e., color-coded duplex ultrasound, we found a relationship between hemodynamic changes (elevated mean blood flow velocities) in the vessels most frequently associated to a HFS (AICA and PICA) and the side of the HFS. This seems to reflect local hemodynamic changes of the vessel as neither the vertebral arteries (diameter, blood flow velocities, resistance index) nor the basilar artery (blood flow velocities, Doppler spectrum, resistance index) were affected. This blood flow velocity elevation might be due to dolicho-ectasia of these vessels, which is reported to be as high as $94.4 \%$ or to their predominance: AICA $90 \%$; PICA $76 \%$ [11]. Furthermore, in our study, analysis of the flow Doppler spectrum and the resistance indices (RI, Table 1), speak against the presence of stenoses.

However, our study has some limitations. The number of patients studied was small; MRA sequences (3D-TOF), allowing a comparison, were not available; the PICA and AICA, due to well-known technical limitation of transcranial ultrasound (attenuation of the signal through the skull, depth of examination) could not be examined along their whole course; and a single sonographer examined all the patients preventing any inter-examiner comparison.

\section{Conclusions}

Despite the limited number of HFS patients studied, we found a significant association between elevation of the blood flow velocities of the PICA and AICA and the side of HFS. HFS seems not only to relate to an unfortunate "malposition" of an artery over the root exit zone of the facial nerve but also to hemodynamic changes detectable by CDFI. Ultrasound techniques may become an additional tool in the detection and evaluation of the "vascular-nerve" conflict of HFS.

Acknowledgments We thank Professor Roger Graves University of Victoria, Canada, for his statistical advice.

Conflict of interest The authors report no conflict of interest concerning the material and methods used in this study or the findings specified in this paper.

\section{References}

1. Auger RG, Whisnant JP (1990) Hemifacial spasm in Rochester and Olmsted County, Minnesota, 1960 to 1984. Arch Neurol 47:12331234

2. Barker FG II, Bissonette DJ, Jannetta PJ, Jho HD, Larkins MV, Shields PT (1995) Microvascular decompression for hemifacial spasm. J Neurosurg 82:201-210

3. Babinski J (1905) Hémispasme facial périphérique. Nouv Iconogr Salpétrière 18:418-423

4. Jannetta PJ (1990) Cranial rhizopathies. In: Youmans JR (ed) Neurological surgery, vol 41, 3rd edn. W.B. Saunders, Philadelphia, pp 4169-4182

5. De Tribolet N, Magistris MR, Sauvain MO (2001) Microvascular decompression of the facial nerve. Oper Tech Neurosurg 4:127-136

6. Magistris MR, Pinelli P, Rillet B, Roth G (1990) Cryptogenic hemifacial spasm. A neurophysiological study. Electromyogr Clin Neurophysiol 30:361-370

7. Kaps M, Seidel G, Bauer T, Behrmann B (1992) Imaging of the intracranial vertebrobasilar system using color-coded ultrasound. Stroke 23:1577-1582

8. Bonafe A, Brunet E, Holley P, Manelfe C, Simonetta-Moreau M (1996) Contribution of time-of-flight magnetic resonance angiography for exploring neurovascular compression (hemifacial spasm or trigeminal neuralgia). J Neuroradiol 23:149-156

9. Chang JH, Chang JW, Chung SS, Han IB, Huh R (2009) Unusual causes and presentations of hemifacial spasm. Neurosurgery 65:130 137

10. Cardoso F, Oliveira LD, Vargas AP (1999) Hemifacial spasm and arterial hypertension. Mov Disord 14:832-835

11. Holley P, Bonafe A, Brunet E, Simonetta-Moreau M, Manelfe C (1996) The contribution of "time-of-flight" MRI angiography in the study of neurovascular interactions (hemifacial spasm and trigeminal neuralgia). J Neuroradiol 23:149-156 